ENERGY TRANSMISSION AND DISTRIBUTION Power theft in India Energy Policy 93, 127-136 (2016)

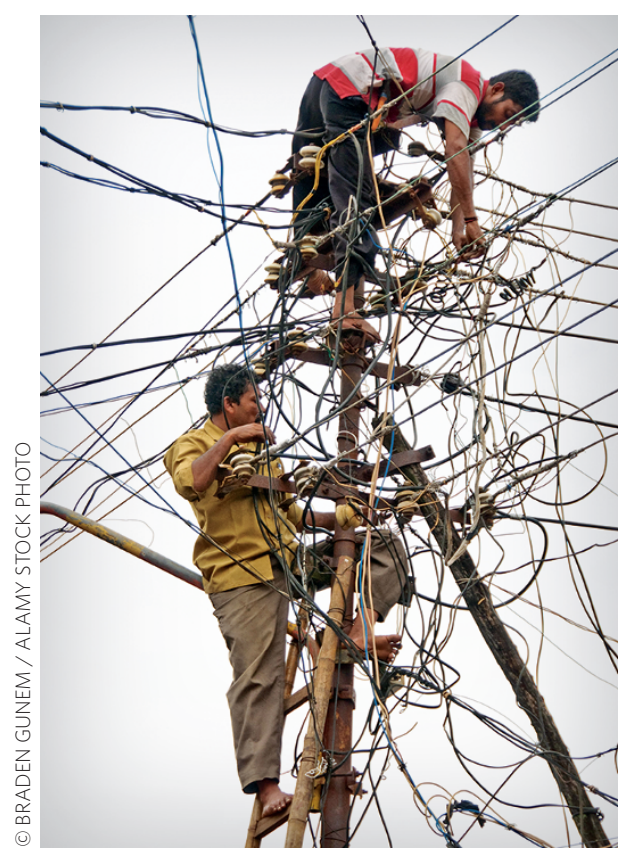

On two successive days in July 2012, India experienced massive power shortages. The second is the largest blackout ever recorded, affecting nearly 700 million people in 22 out of 28 of India's states. Lack of available capacity, weak infrastructure and poor electricity supply governance are recognized as the main causes for such a generalized failure. With regard to governance, it has been suggested that more than $20 \%$ of the total electricity generated in India is stolen. In the first study conducted at the all-India level, Vasundhara Gaur and Eshita Gupta from TERI University have now determined the level of theft in Indian states and found that the causes of power theft are linked to socioeconomic and governance factors.

The study analysed annual data on transmission and distribution losses, obtained from the Central Electricity Authority, for the period 2005 to 2009 . The average value of transmission and distribution losses was over $34 \%$ of the total output, with wide variations among states. Higher levels of corruption and pandering populism of politicians were found to increase power theft, while efficient bill collection reduced it significantly, indicating that more effective management at utility level can help to lessen the problem. These results confirm that top-down policies aimed at achieving lower corruption and better management standards might significantly curb power theft.

\section{WATER OXIDATION}

\section{Two metals better than one} J. Am. Chem. Soc. 138, 3623-3626 (2016)

Electrolysing water allows electrical energy to be stored as chemical energy in the form of molecular hydrogen. For the process to be sustainable, water must be oxidized simultaneously to oxygen in the half-cell reaction at the anode of the electrolyser. Catalysts based on porphyrin molecules ring-shaped biological pigments in which metal ions typically sit in the centre - have been investigated for this oxygen evolution reaction (OER), but relatively few examples exist where two different metal ions are incorporated into the structure. Now, Doris Grumelli at INIFTA in Argentina and colleagues in Germany and Switzerland have prepared porphyrin-based structures containing two different metals, in some cases finding that they are almost two orders

\title{
BATTERIES
}

Chloride-ion batteries are one of the latest additions to the rechargeable battery family. In contrast to lithium-ion batteries in which lithium ions move between electrodes, chloride-ion batteries rely on chloride ion $\left(\mathrm{Cl}^{-}\right)$transport, offering high theoretical energy capacity that rivals their lithium counterparts. However, research into $\mathrm{Cl}^{-}$batteries is still in its infancy, and the current challenge lies in finding suitable electrodes and electrolytes that can offer high cyclability. Maximilian Fichtner and colleagues in Germany have now reported a $\mathrm{Cl}^{-}$battery consisting of a vanadium oxychloride cathode $(\mathrm{VOCl})$, a lithium anode and a solution of 1-butyl-1-methylpiperidinium chloride $\left(\mathrm{PP}_{14} \mathrm{Cl}\right)$ in propylene carbonate as an electrolyte, which displays high stability over 100 cycles.

The researchers revealed that upon discharge, the $\mathrm{PP}_{14}{ }^{+}$ions diffuse into the $\mathrm{VOCl}$ cathode, expanding the $\mathrm{VOCl}$ interlayers, while the vanadium ions are partially reduced to $\mathrm{V}^{+}$; meanwhile, the $\mathrm{Cl}^{-}$ions diffuse into the lithium anode, which is then oxidized into $\mathrm{LiCl}$. While charging, $\mathrm{V}^{2+}$ can be oxidized back into $\mathrm{V}^{3+}$; importantly, the enlarged $\mathrm{VOCl}$ interlayers due to the intercalated $\mathrm{PP}_{14}{ }^{+}$ions allow the return of $\mathrm{Cl}^{-}$into the cathode. These multistep reactions ensure good reversibility during the discharge/charge process in the battery.

of magnitude more active for OER than related structures containing just one metal ion per porphyrin unit.

The researchers prepared monolayers of metal-porphyrin complexes on $\mathrm{Au}(111)$ surfaces. In each structure, two sites can bind metal ions - one in the centre of the molecule and one on the outside of the ring, which is bound by a total of four porphyrin molecules. In one example where $\mathrm{Fe}^{2+}$ ions were in the centre of the porphyrin ring, the addition of $\mathrm{Co}^{2+}$ ions as a secondary metal increased the amount of oxygen evolved by a factor of 86 . Switching the positions of the metals decreased the activity significantly, highlighting the importance of the specific coordination environment of the metal centres.

\section{SILICON SOLAR CELLS}

Titania lets electrons through Sol. Energy Mater. Sol. Cells 150, 32-38 (2016)

Silicon solar cells have by far the largest market share of the photovoltaics (PV) industry. Although silicon PV is a relatively mature technology, continuous advances in materials, device architecture and processing keep pushing their power conversion efficiency (PCE) up and driving costs down. A strategy for increasing the PCE of the best performing silicon cells, based on a heterojunction of crystalline and amorphous silicon, is to use carrierselective contacts, that is, contacts that only let through either electrons or holes. Now, Xinbo Yang and colleagues at the Australian National University have reported a silicon heterojunction cell with an ultrathin $\mathrm{TiO}_{x}$ layer acting as an electron-selective contact that achieves a PCE of about $20 \%$ with simplified, cheaper processing than in the case of amorphous silicon contacts.

A layer of $\mathrm{TiO}_{x}$ is deposited by atomic layer deposition on the back surface of a $2 \times 2 \mathrm{~cm}^{2} \mathrm{n}$-type silicon solar cell. The $\mathrm{TiO}_{x}$ layer passivates the silicon surface, thus reducing carrier recombination, while allowing a relatively low contact resistance of the $\mathrm{TiO}_{x} / \mathrm{n}$-Si heterojunction. The insertion of a $1.2 \mathrm{~nm} \mathrm{SiO}$ layer between a $3.5 \mathrm{~nm} \mathrm{TiO}_{x}$ layer and the silicon improves the thermal stability up to $350^{\circ} \mathrm{C}$, while maintaining good passivation properties. The researchers demonstrate a maximum PCE of $20.5 \%$. Although this value is lower than those reported with selective contacts based on amorphous silicon, the improved thermal stability and simplicity in the fabrication process might make the $\mathrm{TiO}_{x}$ contacts an appealing alternative.

Written by Elisa De Ranieri, James Gallagher, Alessandro Rubino and Changjun Zhang. 